\section{SANDIA REPORT}

SAND97-3111 • UC-402, 403, 406 Unlimited Release

Printed December 1997
RECFIVED

JAN 291998

OSTI

\title{
Photoconductive Semiconductor Switches: Laser Q-switch Trigger and Switch-Trigger Laser Integration
}

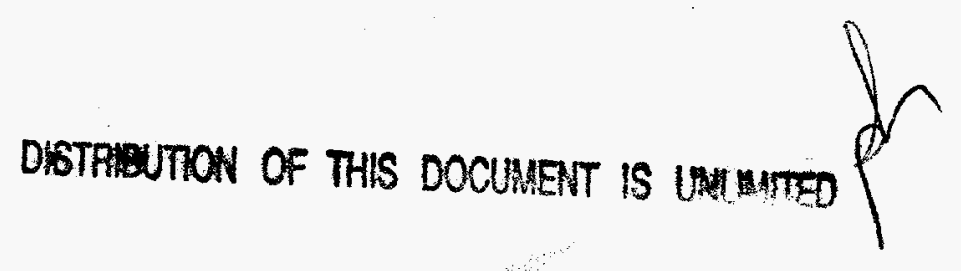

G. M. Loubriel, A. Mar, R. A. Hamil, F. J. Zutavern, W. D. Helgeson

Prepared by

Sandia National Laboratories

Albuquerque, New Mexico 87185 and Livermore, California 94550

Sandia is a multiprogram laboratory operated by Sandia Corporation, a Lockheed Martin Company, for the United States Department of Energy under Contract DE-AC04-94AL85000.

Approved for public release; further dissenination unlimited. 3)

Gindia National Laboratories
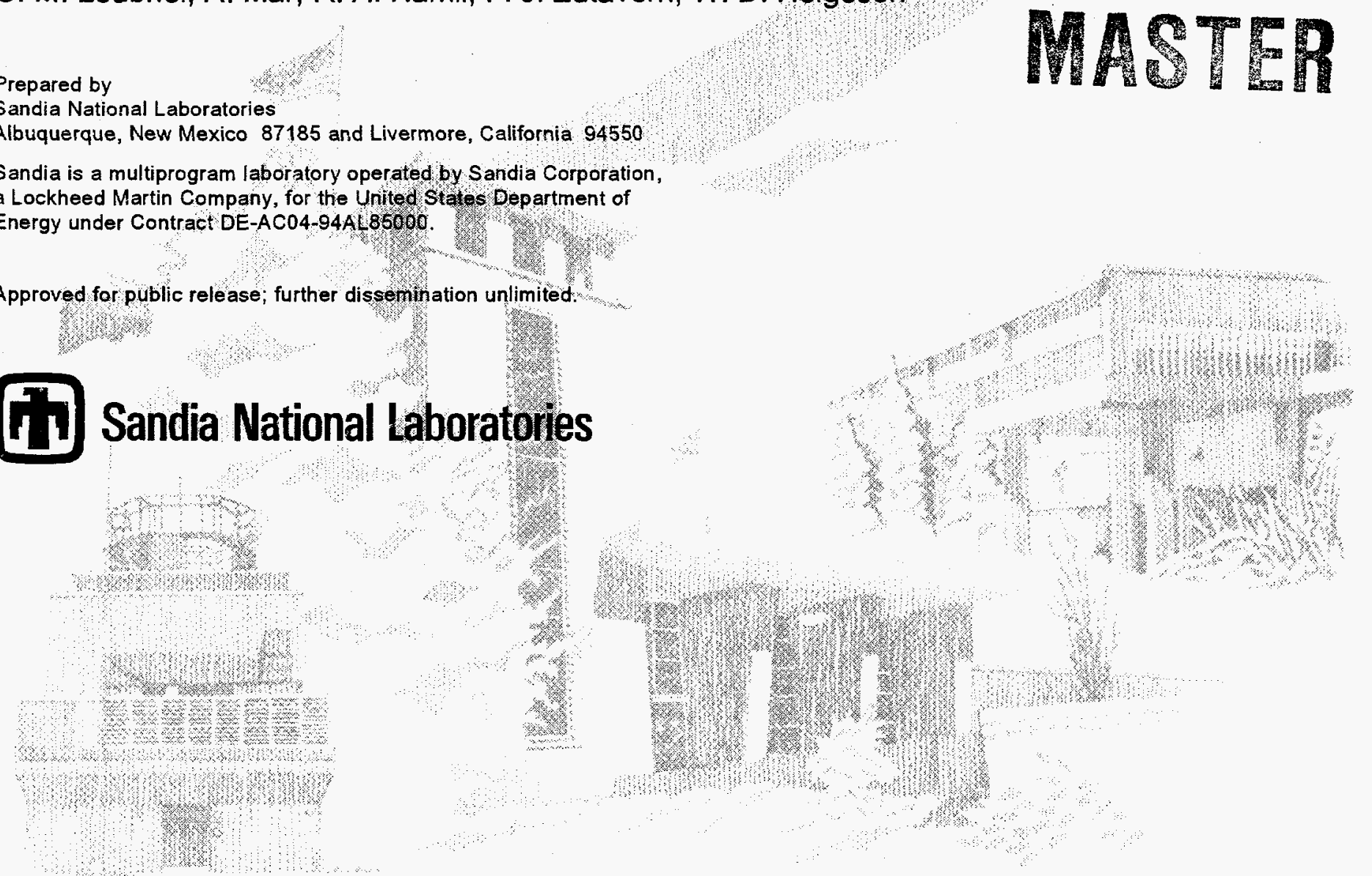


\section{DISCLAIMER}

Portions of this document may be illegible electronic image products. Images are produced from the best available original document. 
SAND 97-3111

Unlimited Release

Printed: December, 1997

\title{
Photoconductive Semiconductor Switches: Laser Q-switch Trigger and Switch-Trigger Laser Integration
}

\author{
G. M. Loubriel, A. Mar, R. A. Hamil, \\ F. J. Zutavern, and W. D. Helgeson \\ High Power Electromagnetics Department \\ Sandia National Laboratories \\ P. O. Box 5800 \\ Albuquerque, NM 87185-1153
}

\begin{abstract}
This report provides a summary of the Pulser In a Chip 9000-Discretionary LDRD. The program began in January of 1997 and concluded in September of 1997. The over-arching goal of this LDRD is to study whether laser diode triggered photoconductive semiconductor switches (PCSS) can be used to activate electro-optic devices such as Q-switches and Pockels cells and to study possible laser diode/ switch integration. The PCSS switches we used were high gain GaAs switches because they can be triggered with small amounts of laser light.

The specific goals of the LDRD were to demonstrate: 1) that small laser diode arrays that are potential candidates for laser-switch integration will indeed trigger the PCSS switch, and 2) that high gain GaAs switches can be used to trigger optical Q-switches in lasers such as the lasers to be used in the X-1 Advanced Radiation Source and the laser used for direct optical initiation (DOI) of explosives. The technology developed with this LDRD is now the prime candidate for triggering the $\mathrm{Q}$ switch in the multiple lasers in the laser trigger system of the X-1 Advanced Radiation Source and may be utilized in other accelerators. As part of the LDRD we developed a commercial supplier. To study laser/switch integration we tested triggering the high gain GaAs switches with: edge emitting laser diodes, vertical cavity surface emitting lasers (VCSELs), and transverse junction stripe (TJS) lasers. The first two types of lasers (edge emitting and VCSELs) did activate the PCSS but are harder to integrate with the PCSS for a compact package. The TJS lasers, while easier to integrate with the switch, did not trigger the PCSS at the TJS laser power levels we used. The PCSS was used to activate the Q-switch of the compact laser to be used in the X-1 Advanced Radiation Source.
\end{abstract}


CONTENTS

$\underline{\text { Section }}$

1.0 SUMMARY 5

1.1 GOALS AND TASKS . 5

1.2 RESULTS 5

2.0 INTRODUCTION: HIGH GAIN GaAs SWITCHES AND

LASERS FOR ACCELERATORS 7

2.1 HIGH GAIN GaAs SWITCHES

2.2 LASERS FOR ACCELERATORS 11

3.0 TRIGGERING POCKELS CELLS AND

OPTICAL Q-SWITCHES IN LASERS 12

3.1 POCKELS CELL ACTIVATION WITH PCSS 12

3.2 ELECTRICALLY Q-SWITCHING THE X-1 LASER 13

3.3 PCSS DRIVER FOR THE X-1 LASER 14

3.4 JTTER IN THE X-1 LASER 17

4.0 LASER SWITCH INTEGRATION 19

4.1 EDGE EMITTING LASER DIODES 19

4.2 VERTICAL CAVITY SURFACE EMITTING LASERS 21

4.3 TRANSVERSE JUNCTION STRIPE LASERS 24

5.0 COMMERCIAL SUPPLIER AND CONCLUSIONS 26

$\begin{array}{ll}\text { 6.0 REFERENCES } & 27\end{array}$ 


\subsection{SUMMARY}

This section summarizes the goals of this project, the tasks required to meet the goals as outlined in the original LDRD proposal, and the results of the LDRD. Section 2 is an introduction that explains what are Photoconductive Semiconductor Switches (PCSS) and the need for lasers in high voltage accelerators. Section 3 of this report covers the use of PCSS in triggering the Q-switch of a compact laser. Section 4 of the report discusses laser/switch integration and, in particular, the use of edge emitting laser diodes, vertical cavity surface emitting lasers (VCSELs), and transverse junction stripe (TJS) TJS lasers to trigger the PCSS. Finally, in section 5 we discuss the development of this technology in the commercial sector.

\subsection{GOALS AND TASKS}

The goals of the LDRD were to demonstrate: 1) that small laser diode arrays that are potential candidates for laser-switch integration will indeed trigger the PCSS switch, and 2) that high gain GaAs switches can be used to trigger optical Q-switches in lasers such as the lasers to be used in the X-1 Advanced Radiation Source and the laser used for direct optical initiation (DOI) of explosives. The technology developed in this LDRD is the prime candidate for triggering the $\mathrm{Q}$ switch in the multiple lasers in the laser trigger system of the X-1 Advanced Radiation Source. As part of the LDRD we were to develop a commercial supplier.

The tasks, as presented in the original proposal, were:

\section{Task/Milestones:}

Planned Actual

1. Demonstrate that the high gain PCSS can properly activate the optical Q-switch of the laser for the X-1 accelerator.

2. Demonstrate the capability of VCSELs to trigger high gain PCSS switches for parameters compatible with task 1.

$5 / 97 \quad 8 / 97$

3. Demonstrate the capability of edge emitting lasers to trigger high gain PCSS switches for parameters compatible with task 1.

4. Develop commercial supplier that will market the Q-switch driver. 7/97

5. Final SAND report.

9/97

$3 / 97 \quad 2 / 97$

$4 / 97 \quad 5 / 97$

$3 / 97$

$12 / 97$

\subsection{RESULTS}

Task 1-- This task is the most crucial one in this LDRD since it impacts the laser trigger system of the X-1 Advanced Radiation Source. Prior to receiving the laser in August of 1997, we demonstrated electro-optic control of a Pockels cell with a PCSS switch. This demonstration was performed by charging a cable and discharging it to the Pockels cell via the PCSS. When we received the laser from AlliedSignal we demonstrated that its Q-switch could be triggered with a high voltage pulser based on a high gain GaAs PCSS. In particular, the optical system jitter was reduced with the high gain GaAs based pulser. The triggering of 
this laser was done using a fiber optic delivery system which is compatible with the X-1 accelerator.

Task 2-- We demonstrated that high power VCSELs are capable of triggering a high gain PCSS. This is a significant result because it demonstrates that the PCSS can be triggered with the types of optical output of micro lasers which may be monolithically integrated with the PCSS. It is also important since: 1) the PCSS was triggered with a record low energy of $2.1 \mathrm{~nJ}, 2)$ the VCSEL is $75 \mu \mathrm{m}$ by $75 \mu \mathrm{m}(5600 \mu \mathrm{m} 2)$ in size with an active area of about $3200 \mu \mathrm{m}^{2}$, and 3) the price of VCSELs is significantly smaller than that of edge emitting lasers. The PCSS discharged a transmission line charged to $3.5 \mathrm{kV}$, producing a $45 \mathrm{~A}$ pulse of about 3 ns duration. The peak optical energy density produced by the VCSEL is 0.65 $\mathrm{J} / \mathrm{m}^{2}$.

Task 3--- We have, in the past, triggered high gain PCSS with edge emitting lasers. In this LDRD we wanted to trigger the switches with edge emitting lasers that are compatible with monolithic integration of switch and laser. Two options were originally considered: using existing transverse junction stripe (TJS) lasers (preferred) or using edge emitting lasers with waveguides. Because of the difficulty in obtaining lasers with waveguide structures in a short amount of time, we tested the TJS lasers and they did not trigger the PCSS.

Task 4- Two companies have expressed an interest in using high gain, optically triggered, GaAs switches for driving Q-switches and Pockels cells in lasers. The president of one of these companies that is a manufacturer of electro-optic modulators and Q-switches; ultra-high speed, high voltage pulse generators; Q-switch drivers; high-speed electro-optic switching systems; and electronics for modulators among other products visited Sandia to study this system and discuss future options. They are very interested in the Q-switching application we demonstrated, especially if the switch longevity is sufficient for marketing it as a product. Another application is in sensor systems based on Optical Parametric Amplifier (OPA). A PCSS driven Q-switch could precisely time the OPA's high energy probe laser. 


\subsection{INTRODUCTION: HIGH GAIN GaAs SWITCHES AND LASERS FOR ACCELERATORS}

\section{$2.1 \quad$ HIGH GAIN GaAs SWITCHES}

High gain PCSS offer switching improvements in voltage, current, rise time, jitter, optical activation, size, and cost. High voltage operation of conventional (linear) PCSS, is limited by optical trigger energy requirements, which are 1,000 to 100,000 times greater than those for high gain PCSS. To understand and develop high gain PCSS many experiments have been performed (for general references, see reference 1). In order to explain what are PCSS and to describe the state-of-the-art, the following paragraphs provide a brief description of some of these associated properties and issues: (1) general device description, (2) high gain, (3) current filaments, and (4) device longevity.

Device Description: The GaAs switches used in this experiment are lateral switches (see figure 2.1) made from undoped GaAs of high resistivity $>10^{7} \Omega$-cm and metallic lands that connect the switch to an energy source and a load. The metallic contacts provide either $\mathrm{p}$ or $\mathrm{n}$ doped regions. The simplest $\mathrm{n}$ contact is the ubiquitous Ni-Ge-Au-Ni-Au metallization. The $\mathrm{p}$ contacts are made from Au-Be. The insulating region separating the two contacts (the gap, in analogy to spark gaps) has a length that varies from $0.2 \mathrm{~mm}$ to $3.4 \mathrm{~cm}$ since higher switched voltages require a larger gap to avoid surface flashover. Because of high electric fields the switches were immersed in a dielectric liquid (Fluorinert ${ }^{\circledR}$ ). Pulse charging of this configuration is typically required to reduce the surface flashover problem. For the tests in this report, a laser diode array was used to trigger the switch. The array consisted of three laser diodes coupled to a $400 \mu \mathrm{m}$ - diameter fiber optic. The array delivered about $1 \mu \mathrm{J}$ in 20 ns at $808 \mathrm{~nm}$ to illuminate the switch.

Since the SNL discovery of a high gain switching mode in GaAs, these switches have been investigated for use in many high voltage applications such as: impulse and ground penetrating radar, switches for firing sets for weapons, as drivers for laser diode arrays to allow detection of objects through fog and smoke, and high voltage accelerators. PCSS offer improvements over existing pulsed power technology. The most significant are: $100 \mathrm{ps}$ risetime, kilohertz (continuous) and megahertz (burst) repetition rates, scalable or stackable to hundreds of kilovolts and tens of kiloamps, optical control and isolation, and solid state reliability. Table I shows the best results obtained with the switches for various applications.

In all the applications listed, the switch has been designed as a stand-alone system with a separate laser that triggers the switch. In this project we demonstrated that small lasers, capable of being monolithically integrated with the switch, did trigger the switch. This development, coupled with recent advances in switch lifetime/longevity, will allow rapid entry into new applications such as $\mathrm{Q}$ switches for lasers as required by many commercial lasers. 


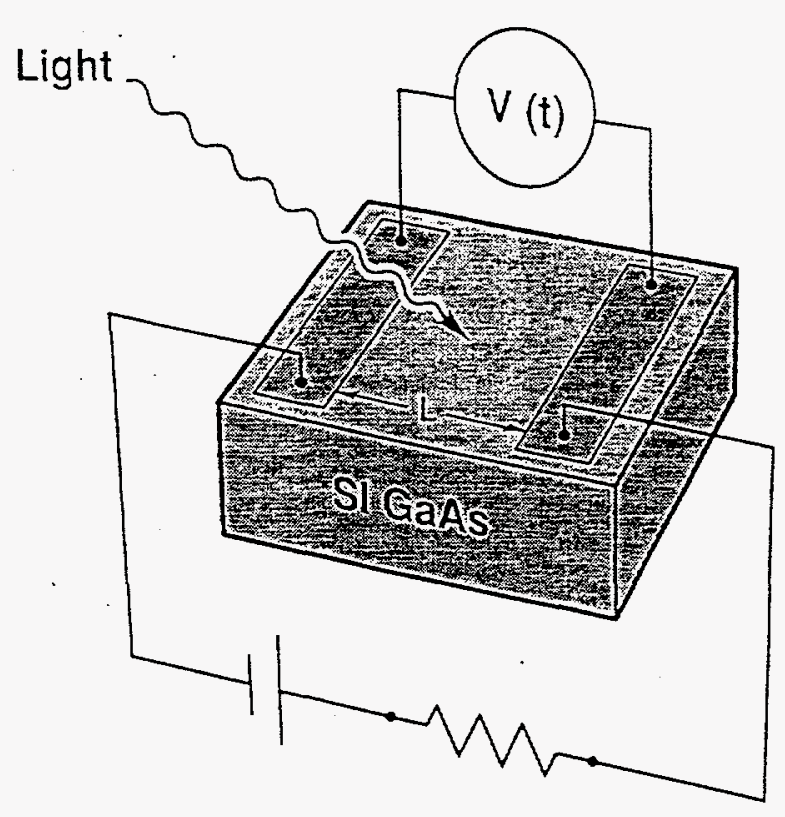

Figure 2.1. Schematic of the lateral semi-insulating (SD GaAs switches used in this study. In this circuit the switch is being used to discharge a capacitor into a resistive load. The switch dimensions depend on the application. The distance between the contacts (the "gap," in analogy to spark gaps) varies from $0.2 \mathrm{~mm}$ to $3.4 \mathrm{~cm}$, for example. Switch thickness (the vertical direction) is always 0.6 $\mathrm{mm}$.

\begin{tabular}{|l|c|c|}
\hline \multicolumn{1}{|c|}{$\begin{array}{c}\text { Table I } \\
\text { Parameter }\end{array}$} & $\begin{array}{c}\text { GaAs, high gain mode, } \\
\text { best individual results. }\end{array}$ & $\begin{array}{c}\text { GaAs, high gain mode, } \\
\text { simultaneous results. }\end{array}$ \\
\hline Switch Voltage (kV) & 200 & 100 \\
\hline Switch Current (kA) & 7.0 & 1.26 \\
\hline Peak Power (MW) & 120 & 48 \\
\hline Rise time (ps) & 350 & 430 \\
\hline R-M-S jitter (ps) & 80 & 150 \\
\hline Optical Trigger Energy (nJ) & $13(2)$ & 180 \\
\hline Optical Trigger Gain & $10^{5}$ & $10^{5}$ \\
\hline Repetition Rate (Hz) & 1,000 & 1,000 \\
\hline Electric Field (kV/cm) & 100 & 67 \\
\hline Device Lifetime (\# pulses) & $>50 \times 10^{6}$ & $5 \times 10^{4},($ at $77 \mathrm{kV})$ \\
\hline
\end{tabular}

Table I. Best switching results for high gain GaAs switches. The numbers reflect the results prior to this LDRD except for the numbers in parenthesis which were demonstrated during this LDRD. 
High Gain: Conventional PCSS produce only 1 electron-hole pair per absorbed photon. The energy of the individual photons excite electrons from the valence band to the conduction band. This excitation is independent of the electric field across the switch, and conventional PCSS can be operated to arbitrarily low voltage. High gain PCSS, on the other hand, occurs only at high electric fields (greater than $4 \mathrm{kV} / \mathrm{cm}$ ). The photo-excited carriers, which are produced by an optical trigger, gain enough energy from the electric field to scatter valence electrons into the conduction band. This process is called impact ionization or avalanche carrier generation. Because many carriers are produced per absorbed photon, switches operating in this mode require extremely low energy optical trigger pulses, and they are called high gain PCSS in contrast to the conventional PCSS, which are often called linear PCSS. To stand off high voltage, PCSS must be made long enough to avoid avalanche carrier generation with no optical trigger (dark breakdown). The optical trigger energy for a linear PCSS scales with the square of its length. So high voltage linear PCSS can require rather high energy optical trigger pulses $(25 \mathrm{~mJ}$ for $100 \mathrm{kV}$ switches switched to $1 \Omega$ ). It is the high gain feature of GaAs PCSS that allows their triggering with small semiconductor LDA. We have triggered $100 \mathrm{kV}$ gallium arsenide (GaAs) PCSS, with as little as $90 \mathrm{~nJ}$. A GaAs PCSS, operating in a low impedance circuit, can produce 100,000 times as many carriers as a linear PCSS would produce.

With most insulating materials (e.g. plastic, ceramic, or glass) and undoped semiconductors (e.g. silicon, gallium phosphide (GaP), or diamond), bulk avalanche carrier generation does not occur below $200 \mathrm{kV} / \mathrm{cm}$. Semiconductors such as $\mathrm{GaAs}$ and indium phosphide (InP) are very different in that high gain PCSS can be initiated at unusually low electric fields (4-6 $\mathrm{kV} / \mathrm{cm}$ and $15 \mathrm{kV} / \mathrm{cm}$, respectively). Surface breakdown limits the field across PCSS to less than the bulk breakdown field (generally $100 \mathrm{kV} / \mathrm{cm}$ ). Since PCSS need to absorb light through a surface, optically-activated avalanche breakdown is only practical in materials which exhibit high gain at lower fields. A very important part of the research into high gain PCSS has been to develop models for high gain at low fields in these materials.

Once avalanche carrier generation is initiated, it continues until the field across the switch drops below a threshold (4-6 kV/cm depending upon the type of GaAs). Since carrier generation causes the switch resistance to drop, in most circuits, the field across the switch will also drop. Indeed, when we first observed high gain PCSS, the most outstanding feature was that at high fields, when the switches would turn on, their voltage would drop to a constant non-zero value and stay there until the energy in the test circuit was dissipated. We originally called this switching mode "lock-on" to describe this effect. "High gain" has been adopted more recently to help distinguish this type of switching mode from other modes which also exhibit persistent conductivity, such as thermal runaway, and single or double injection. The field dependence for avalanche carrier generation that is exhibited in high gain PCSS is similar to that exhibited by Zener diodes. In series with a current limiting resistor, they will conduct whatever current is necessary to maintain a constant voltage across their contacts. In the case of a high gain PCSS, this voltage/field is the "lock-on" voltage/field or the threshold to low field avalanche carrier generation. The phase of switching during which the switch maintains this constant voltage drop is called the sustaining phase, and testing and modeling this phase is also a critical area of our research. 
Current Filaments: Another important feature of high gain PCSS is that the current forms in filaments which are easily observed with a near infra-red sensitive camera (most nonintensified, black and white, CCD-based cameras). When the carriers recombine (i.e. conduction electrons drop back into the valence band), infra-red photons are emitted at approximately $875 \mathrm{~nm}(1.4 \mathrm{eV})$. If the filaments are near the surface of the switch, the emitted photons escape and they can be detected by a camera. Some images obtained in this manner are shown in figure 2.2. We believe that current filaments are fundamental to high gain PCSS and we have never observed high gain without current filaments. While current filamentation can lead to catastrophic destruction of the PCSS, current amplitude and pulse widths can be limited to allow non-destructive operation. In addition, the optical trigger can be distributed across the switch in a manner which creates multiple or diffuse filaments to extend the lifetime or current carrying capacity of the switch.
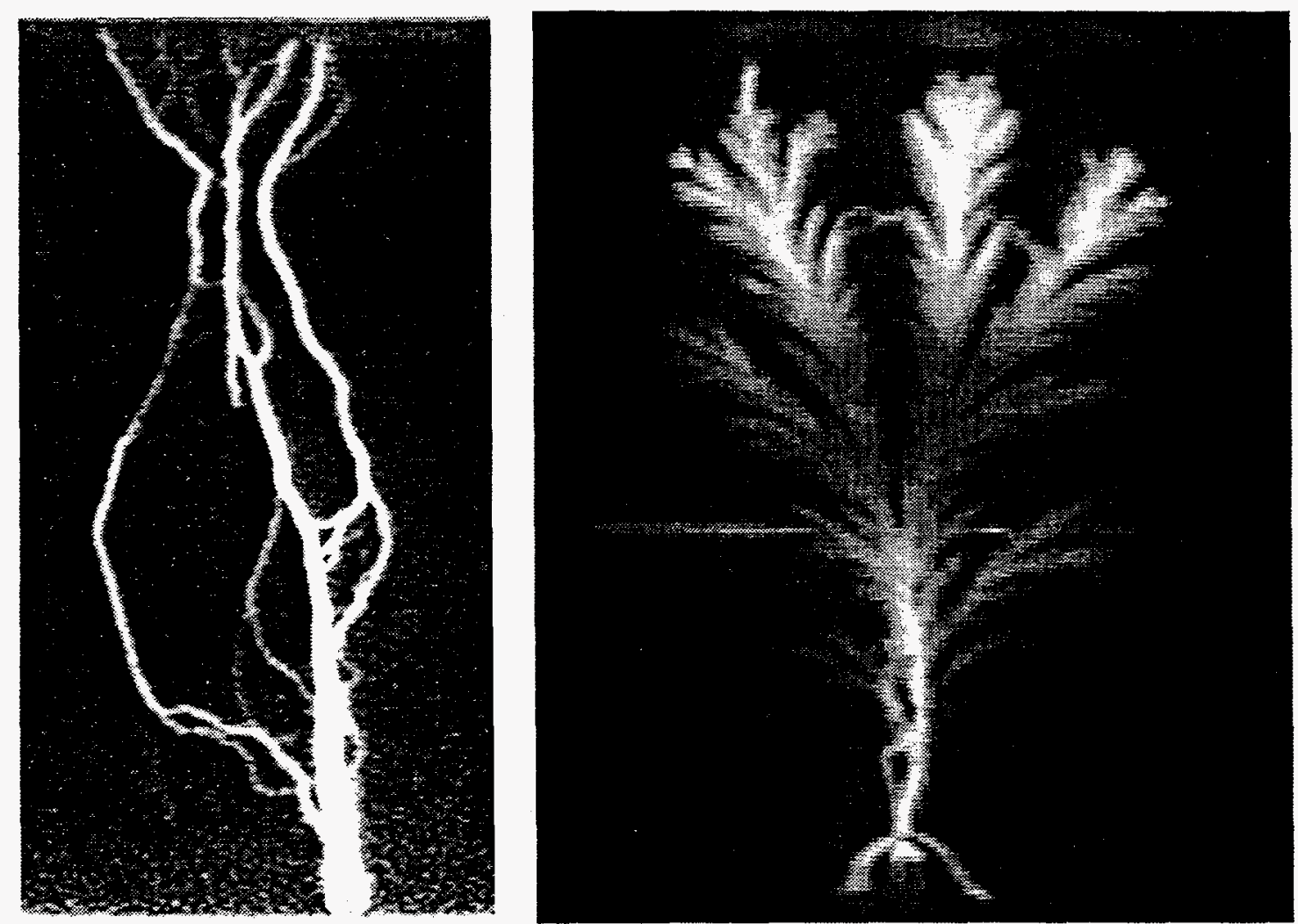

Figure 2.2. These photographs show examples of the current filaments which form during high gain PCSS operation. The images are recorded from the infrared $(875 \mathrm{~nm})$ radiation which is emitted as the carriers recombine in the switch. In both cases, the switches are $1.5 \mathrm{~cm}$ long (vertically). The PCSS on the left was charged to $45 \mathrm{kV}$ and conducted $350 \mathrm{~A}$ for $10 \mathrm{~ns}$. The PCSS on the right was charged to $100 \mathrm{kV}$ and conducted $900 \mathrm{~A}$ for $1.4 \mathrm{~ns}$.

Device Longevity: The biggest problem caused by the filaments is the gradual accumulation of damage at the contacts which limits their useful life to $1-10,000,000$ shots depending upon 
the current per filament and the optical trigger distribution. Presently, device lifetime is a limitation of this technology for some applications. Although there is little change in performance, the PCSS degrade in time because the regions near the contacts are damaged on each pulse, and they gradually erode. The bulk semiconducting material shows very little, if any, degradation as the contact regions wear out. The fact that the degradation is confined to regions near the contacts suggests that substantial increases in switch lifetime can be made by developing better contacts that allow for higher current density. When this project started, high-gain PCSS would last for $\sim 10^{4}$ pulses under a specific set of test conditions ( $\left.0.5 \mathrm{MW}\right)$. During the course of this project, the lifetime has been improved to $5 \times 10^{7}$. In comparison, the semiconductor lasers, which trigger or are driven by these switches, can last from $10^{8}$ to $10^{10}$ pulses. We are presently fabricating new generations of deep-diffused and epitaxialgrown contacts that should yield further improvements. At higher powers or longer pulses, lifetimes are reduced. We plan to address these issues and accelerate lifetime testing by shifting our interests to higher current and higher repetition rate testing. Reference 2 describes our longevity experiments up to June of 1997.

\subsection{LASERS FOR ACCELERATORS}

The $\mathrm{X}-1$ accelerator is being designed to take advantage of the recent scientific breakthroughs that have occurred on the $Z$ accelerator at Sandia in the area of z-pinch physics. X-1 will deliver between 60 and 80 million $A$ to a wire load at the center of the accelerator. In order to deliver such a high current in a period of approximately $100 \mathrm{~ns}$, it is necessary to simultaneously trigger as many as 30 individual accelerator modules which make up X-1. Laser triggering will be required to achieve the necessary synchronization of the 240 highvoltage gas switches in $\mathrm{X}-1$.

The precise firing of multiple accelerator modules in X-1 can be accomplished in two, very different, ways: (1) with one central laser system and an extremely complex optical delivery system, or (2) with a modular laser system using smaller lasers and fewer optics but with the requirement that each laser module be fired with $1 \mathrm{~ns}$ jitter or less. The first approach (1) is presently in use in SNL accelerators such as the $\mathrm{Z}$ accelerator which incorporates a centralized system with 300 optics but for X-1 it is estimated that this type of approach would require more than 5,000 optical elements that would cost millions of dollars. The alignment problems associated with such a system in the high vibration and shock environment of an accelerator make such a system impractically complex and unaffordable. A simpler design (2) involves sending low voltage signals or low energy optical signals to trigger compact lasers at each accelerator. The prime candidate for such a task is a high gain GaAs switch based system. As part of this LDRD study, these GaAs switches have demonstrated the ability to trigger a prototype X-1 laser module with about $1 \mathrm{~ns} \mathrm{rms}$ jitter. There is significant cost savings $(\$ 7 \mathrm{M})$ in taking the modular approach to the trigger system which is half the cost of the central laser approach. 


\subsection{TRIGGERING POCKELS CELLS AND OPTICAL Q-SWITCHES IN LASERS}

\subsection{POCKELS CELL ACTIVATION WITH PCSS}

An important application of high gain PCSS is in the production of fast voltage risetimes that control an electro-optic modulator such as a Pockels cell or a Q-switch. In a previous experiment (Reference 3) we placed a Pockels cell in the output of a Nd:YAG (1064 nm) laser. The laser pulse had a duration of about $30 \mathrm{~ns}$. We demonstrated electro-optic modulation by allowing only half of the laser pulse to be transmitted. The crucial parameter is the risetime of the off to on transition which we want to reduce to below one nanosecond. Electrically, the Pockels cell is a capacitor of about $30 \mathrm{pF}$. The circuit we implemented charges this capacitance and keeps it charged while the first part of the laser pulse enters the Pockels cell. Then the high gain PCSS is activated and the switch shorts the capacitance of the Pockels cell. The current risetime determines the risetime or fall-time of the optical transition. In that work we were able to obtain an optical signal with a risetime of 600 ps.

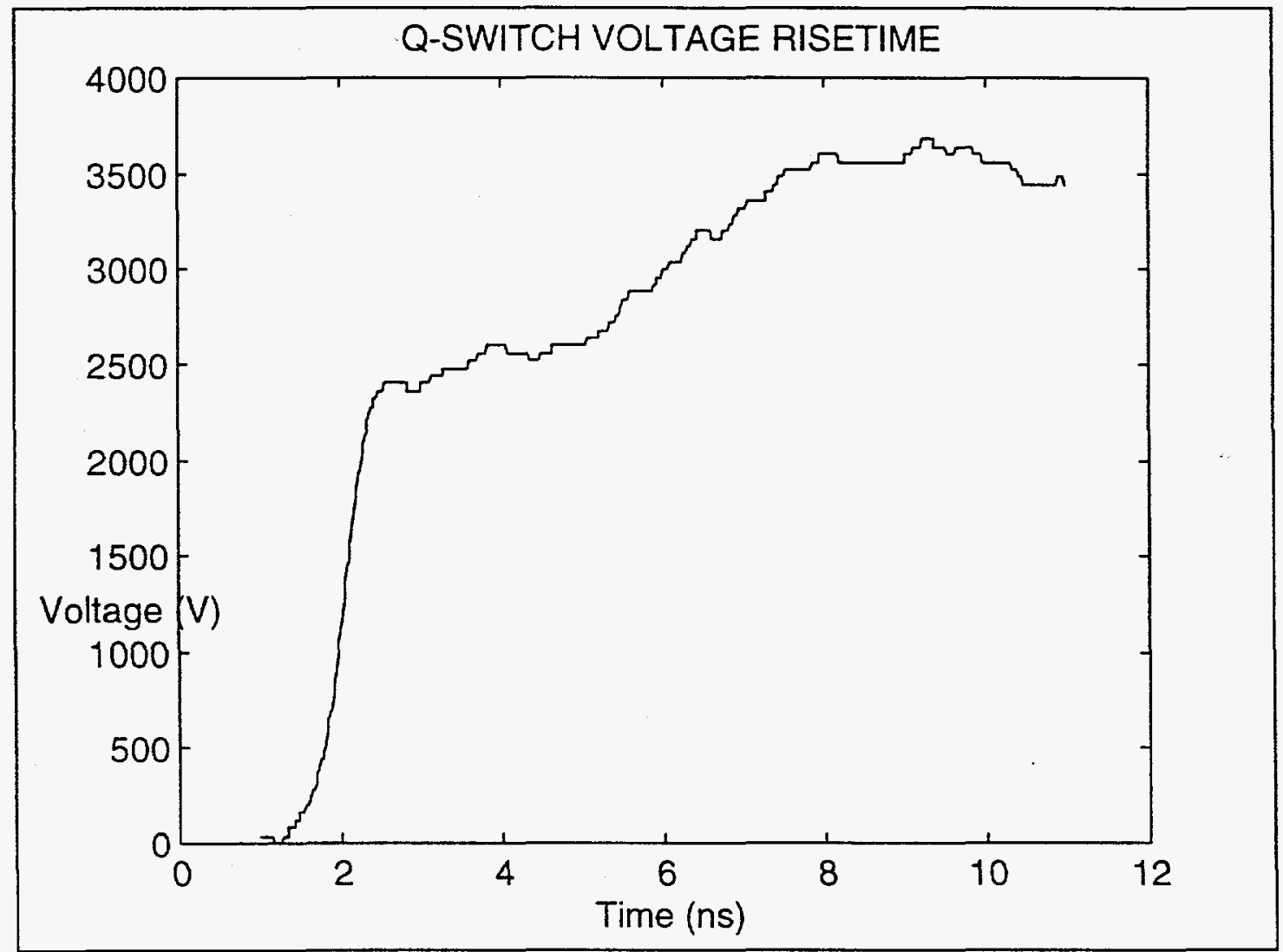

Figure 3.1. The voltage across the Pockels cell. 
The problem with that circuit is that the Pockels cell capacitance was charged and then discharged. In this work we wanted to demonstrate that we could have the Pockels cell at zero voltage and then turned on with a PCSS based circuit. Another problem with the previous work is that the pulsed Nd:YAG laser we used had considerable ringing. For this project we used a CW Diode -pumped Nd:YAG ring laser: Lightwave Electronics Series 120. The Pockels cell voltage waveform is shown in figure 3.1 and the optical waveform in figure 3.2. Note that the risetime of the voltage waveform is slower than that of the optical waveform. The reason for that is that the optical transmission in the system is proportional to the cosine of a term linear in the voltage.

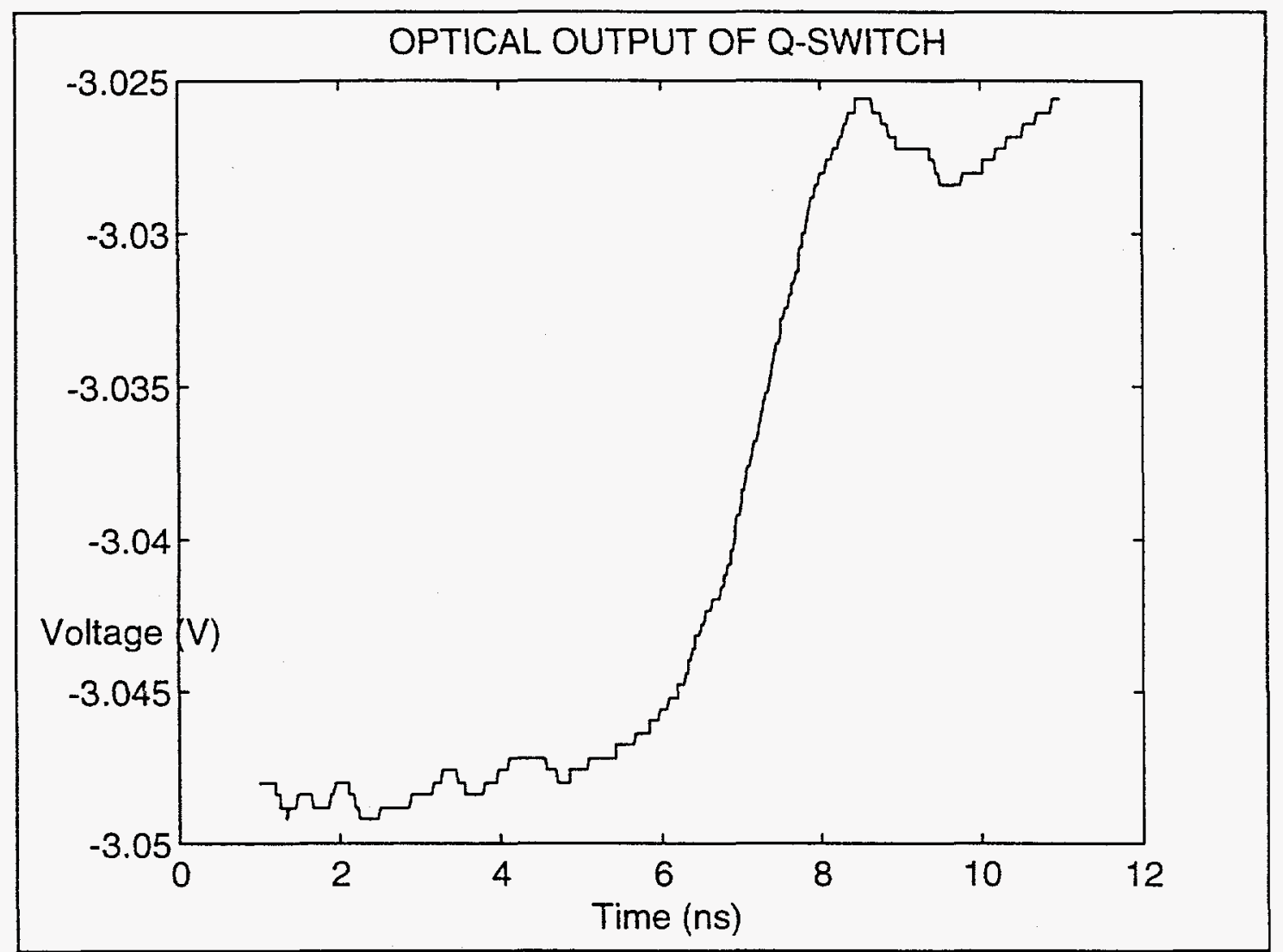

Figure 3.2. Optical waveform with Pockels cell switching. The y axis is not scaled: the optical signal actually drops from full scale (Q-switch off) to zero (Q-switch on) in 2 ns.

\subsection{ELECTRICALLY Q-SWITCHING THE X-1 LASER}

The laser that is being developed to trigger the $\mathrm{X}-1$ modules can be triggered electrically or with the PCSS. We will call this laser the X-1 laser. The X-1 laser, as received, has a very slow electrical system. The risetime of the voltage in the Q-switch is about $100 \mathrm{~ns}$. Figure 3.3 shows the Q-switch voltage and the output of the X-1 laser from multiple pulses to 
emphasize laser jitter. Our intent is to replace this slow Q-switch voltage pulse with a faster pulse power system. Also, the power supply for the X-1 laser generates the voltage pulse which is transmitted electrically to the actual Q-switch upon the arrival of an electrical trigger to the laser's power supply. The PCSS system allows for an optical link between the laser supply and the trigger for the laser.

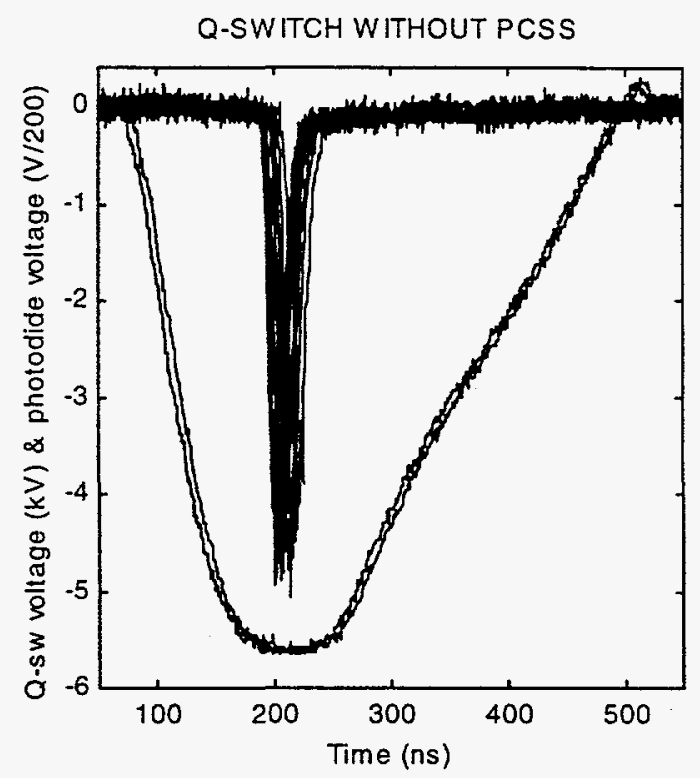

Figure 3.3. Voltage on the Q-switch (2 waveforms) and output laser waveforms (14) of the X-1 laser. The voltage has a risetime of about 150 ns. The laser's output, in the UV, is a 20 ns duration pulse.

\subsection{PCSS DRIVER FOR THE X-1 LASER}

We developed a pulser to charge the Q-switch in the X-1 laser. The circuit is shown in figure 3.4. A $0.1 \mu \mathrm{F}$ capacitor is charged to about $600 \mathrm{~V}$ and discharged (with an SCR) through a step-up transformer. The output of the transformer is then directed to the Q-switch through a diode stack and the PCSS (not shown in figure 3.2). The voltage on the Q-switch is shown in figure 3.5. Figure 3.6 shows a photograph of the overall pulsed power setup, including the power supply for the PCSS, the laser diode array and its electronics, and the PCSS circuit. Figures 3.7 and 3.8 show close-ups of the laser diode array and its electronics (figure 3.7) and the PCSS circuit (figure 3.8). The pulser charges the three capacitors at the top of the photograph and these are discharged through the PCSS (middle left) and two resistors (bottom) to charge the Q-switch. 


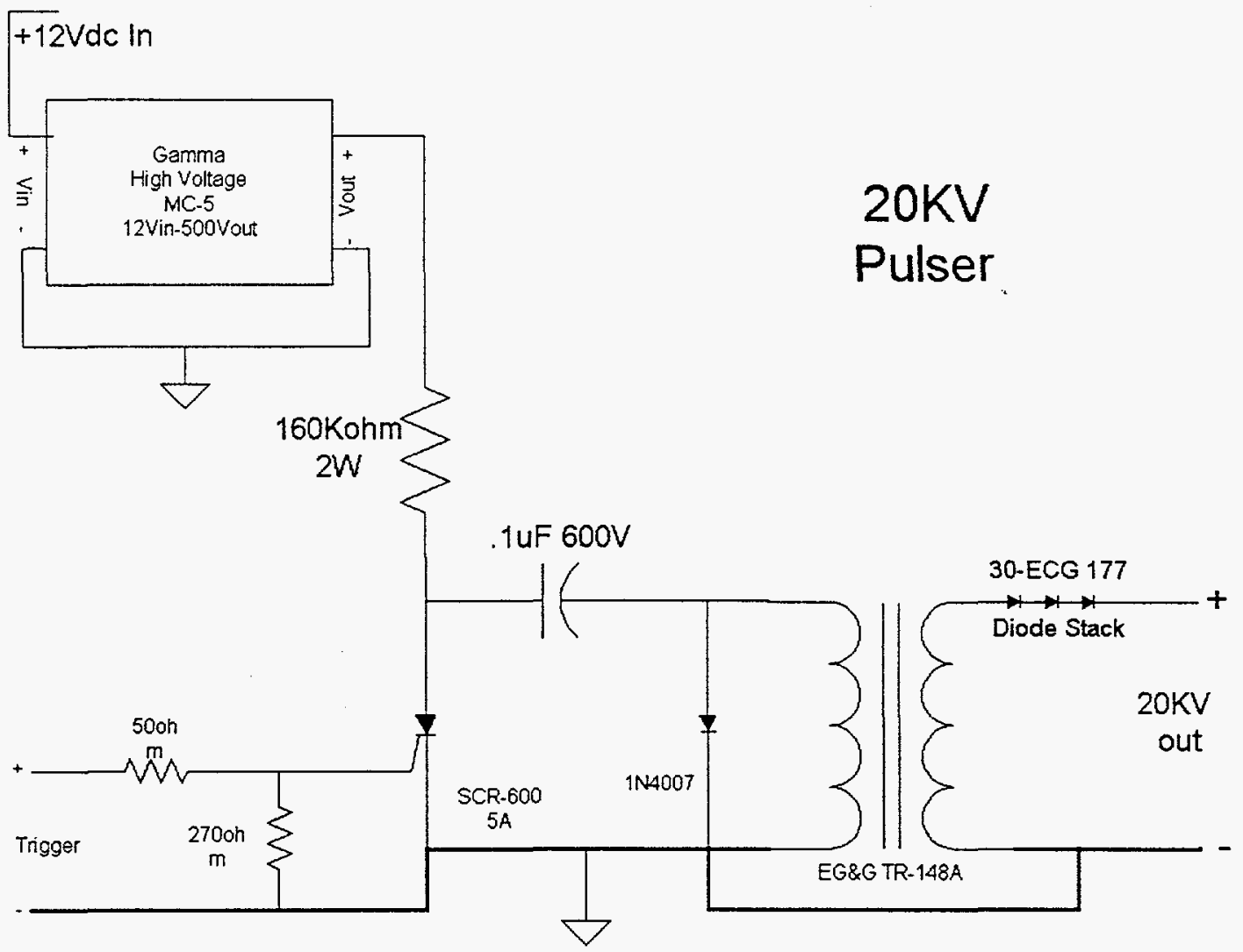

Figure 3.4. Electrical circuit for the pulser that was used to charge the PCSS. The PCSS is in series with the diode stack.

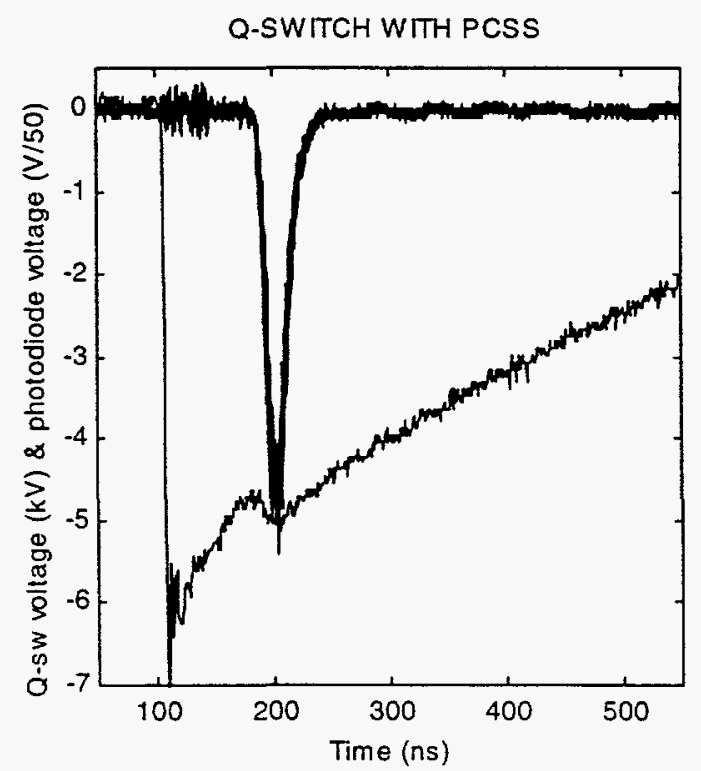

Figure 3.5. Voltage on the Q-switch and output laser waveforms of the X-1 laser. The voltage has a very fast risetime due to the PCSS. 


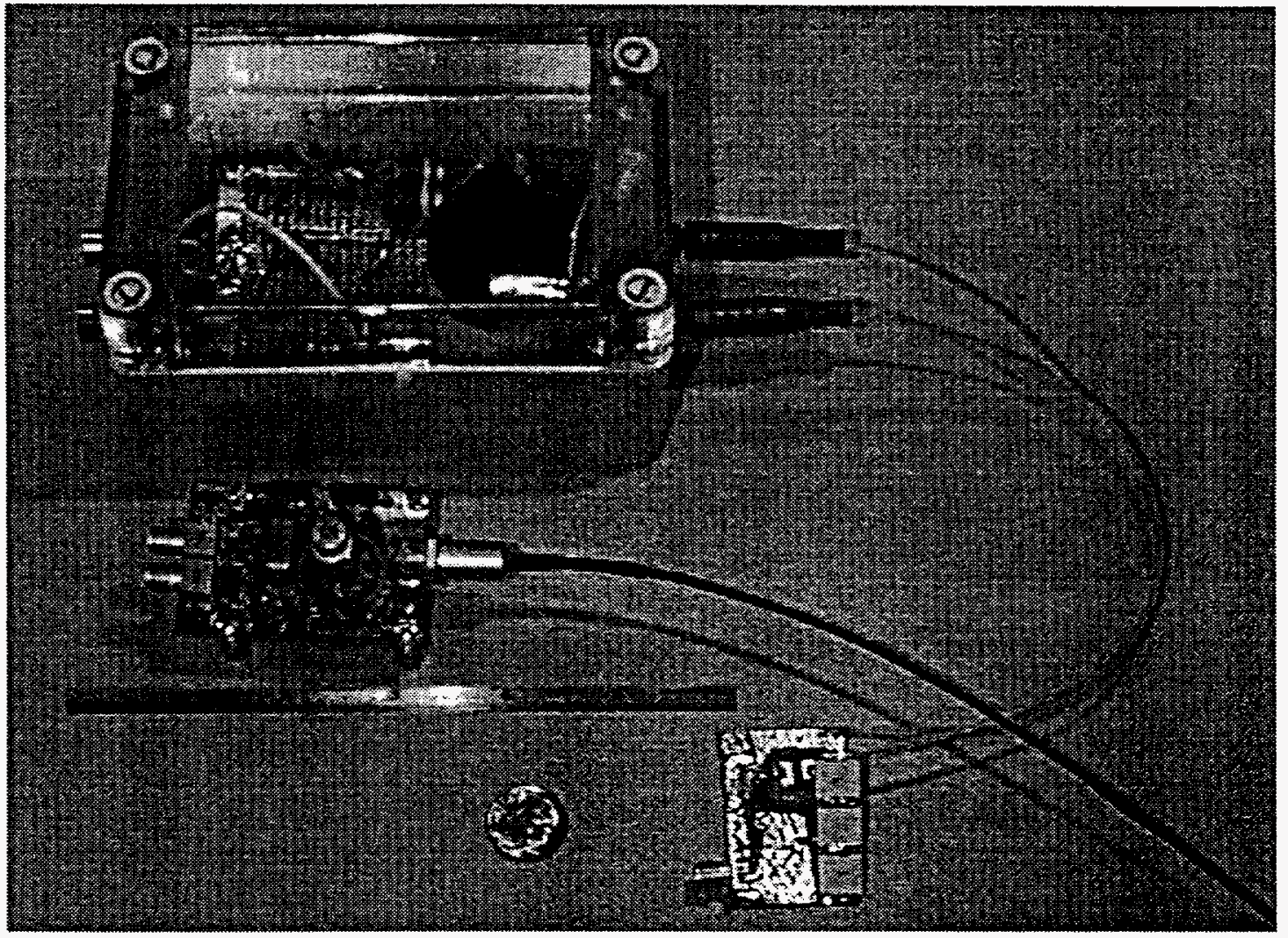

Figure 3.6. This photograph shows, from top to bottom, the power supply for the PCSS, the laser diode array and its electronics, a six inch ruler, a dime, and the PCSS circuit.

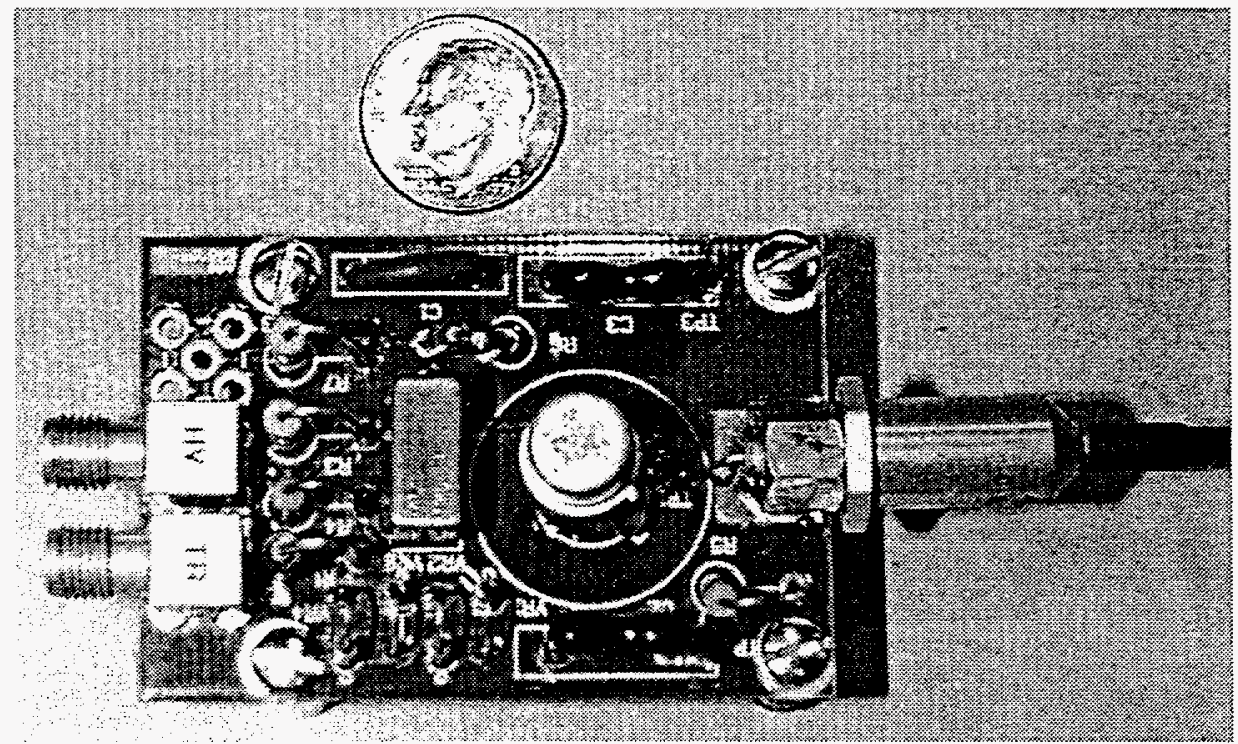

Figure 3.7. This close-up photograph shows the laser diode array, its electronics, and the optical fiber through which the laser's output is taken to the PCSS. The laser diode array and the fiber are to the right. To give an idea of size a dime is also shown. 


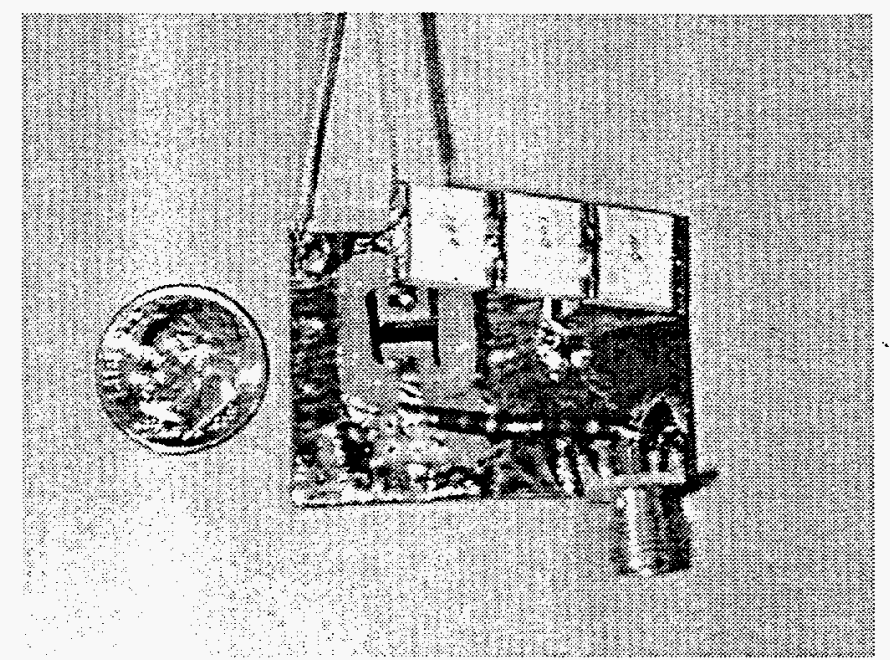

Figure 3.8. This photograph shows the PCSS circuit. The pulser charges the three capacitors at the top of the photograph and these are discharged through the PCSS (middle left) and two resistors (bottom) to charge the Q-switch.
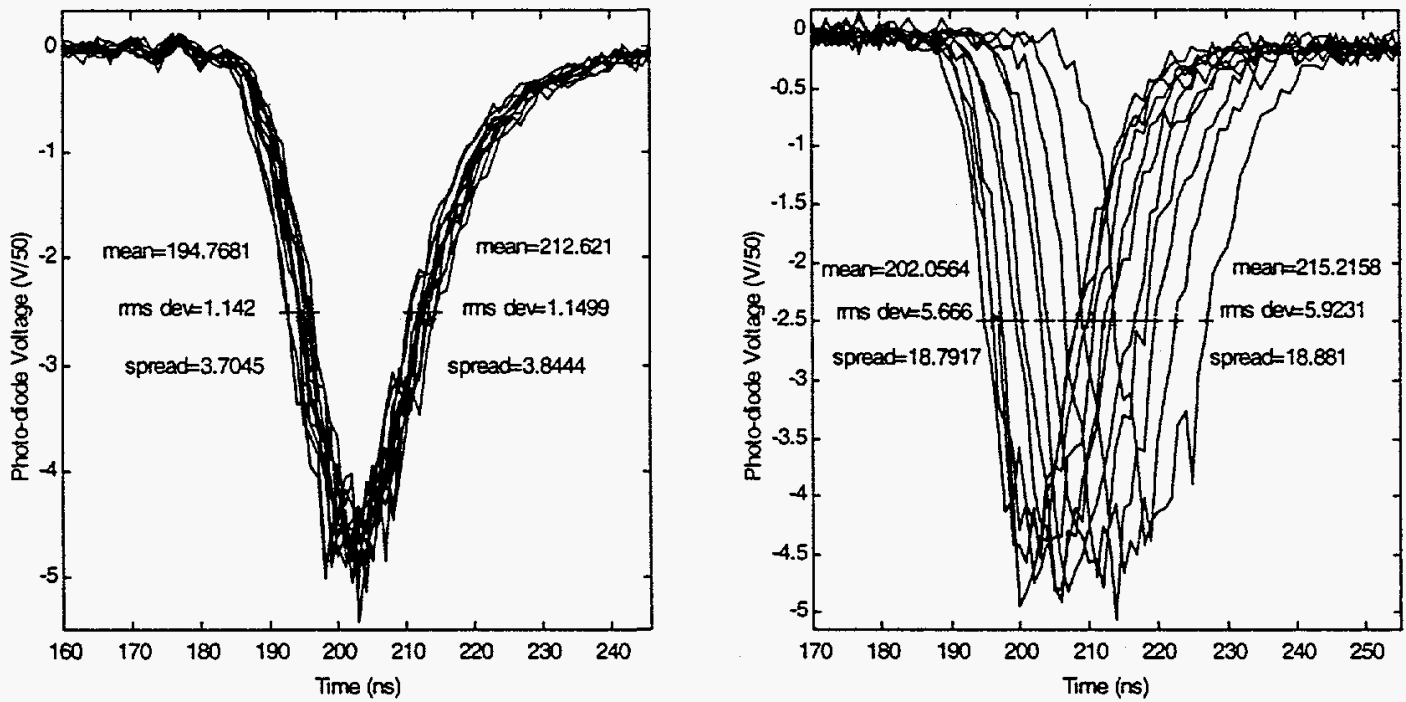

Figure 3.9. The $\mathrm{X}-1$ laser output when the Q-switch is triggered with the PCSS based circuit (left) and with the original electrical circuit (right). There are 13 waveforms superimposed in the panel on the left and 11 on the panel on the right.

\subsection{JITTER IN THE X-1 LASER}

A crucial parameter in the X-1 laser is low jitter. Figure 3.9 shows the laser's output when the laser is Q-switched with the original electrical circuit (right) and with the optically 
isolated PCSS based circuit. The overall laser/ system jitter was reduced from 5.9 ns to 1.1 ns. Another aspect of interest is the jitter inherent in the PCSS, that is, the variation in the delay between the optical trigger pulse and the onset of switching. We measured this jitter with a $1 \mathrm{~mm}$ long GaAs PCSS charged to $4 \mathrm{kV}$. It was triggered with a single laser diode modified for fast rise time with approximately $1 / 2 \mu \mathrm{J}$ of $880 \mathrm{~nm}$ light. A $4 \mathrm{GHz}$ transient digitizer and high speed PCSS circuit was used to make this measurement. The trigger pulses were delivered through a $300 \mu \mathrm{m}$ diameter fiber optic placed near the surface of the switch. Ninety-six successive current waveforms were recorded. The root-mean-square variation was $80 \mathrm{ps}$ and the first-to-last spread was 390 ps. 


\subsection{LASER/SWITCH INTEGRATION}

High voltage photoconductive semiconductor switches (PCSS) have not been integrated with lasers due to the isolation problem that it entails. The main reason is that the switches usually require uniform illumination and the laser structure is a weak link that results in surface flashover. The high gain mechanism we discovered allows for spot illumination near one of the contacts and thus the problem is made less difficult. Another reason why these systems have not been implemented in laser systems is switch longevity. A few years ago these switches would only last for 50,000 pulses at currents of $10 \mathrm{~A}$. That situation has changed considerably and the switch lifetime is now well above $10,000,000$ pulses (where we have stopped measuring). In general there are 3 basic problems with integrating the laser and the PCSS: 1) Field in the vicinity of the PCSS can be as high as $100 \mathrm{kV} / \mathrm{cm}$ which can shift all the bandgap energies in the laser and affect laser performance, 2) Electromagnetic interference of the laser when the PCSS fires, and 3) that the path the light takes to reach the switch can be a weak link such that the current of the PCSS takes this path (to the laser) instead of the intended path (to the load).

That brings us to explain why we want to integrate the switch and the laser to create a high voltage, high speed optoisolator. The advantage of an integrated pulser in a chip are: 1) simplicity of use and reduced labor cost in manufacture of systems because it eliminates the need to align the laser and switch; 2) higher resistance to environmental aspects such as vibration, shock, and temperature (the switch and laser see the same temperature and their bandgaps will shift equally); and 3) longer system life because the exacting alignment of laser and switch will likely increase switch lifetime and require less laser energy. These advantages are added to the advantages of a high gain PCSS: 1) extremely fast high voltage switching with low jitter in a small physical volume; 2) low cost and high reliability; 3) flexibility of implementation in different systems (can be scaled in voltage and current); and 4) further integration of switch with low voltage logic.

\subsection{EDGE EMITTING LASER DIODES}

In the past we have used small edge emitting laser diode arrays to trigger the switches. These were purchased from Laser Diode Inc. (model CVD-167F) and consisted of an array of three laser diodes that are hard coupled to a fiber optic. Their wavelength is around $880 \mathrm{~nm}$. Previous studies at Phillips Lab show that this is the best wavelength to use in terms of the sensitivity of the switches to this light. The pulse duration was $20 \mathrm{~ns}$. The total laser energy in each pulse is measured to be $1.8 \mu \mathrm{J}$ at the end of the $430 \mu \mathrm{m}$ fiber $(90 \mathrm{~W})$. The electronics that power this laser are built at SNL. In this case a single avalanche transistor is switched to deliver a pulse to the array. The package measures $2 "$ by $2 "$ by $1 "$. The package requires a voltage of $400 \mathrm{~V}$ dc and a trigger. We are now using a small box that contains a dc power supply and a trigger generator to provide the inputs to the package. The power supply and trigger generator in the box are respectively about 3 " by 1 " by 1.5 " (most of which is a dc to dc converter powered by $12 \mathrm{~V}$ ) and 3 " by 2 " by 1 ". This laser can be used repetitively at up to $1 \mathrm{kHz}$. Thus, the total size of a package of a laser diode array, the electronics, power supply, and trigger generator is less than 15 cubic inches. 
We have also triggered the high gain switches with single laser diodes. Figure 4.1 shows the optical waveform. The laser energy is $0.15 \mu \mathrm{J}$. This pulse can also be used to trigger a PCSS. Figure 4.2 shows the current and voltage waveforms when a $0.5 \mathrm{~mm}$ long PCSS discharges a $150 \mathrm{nF}$ capacitor charged to $1 \mathrm{kV}$. The current waveform peaks at about $650 \mathrm{~A}$.

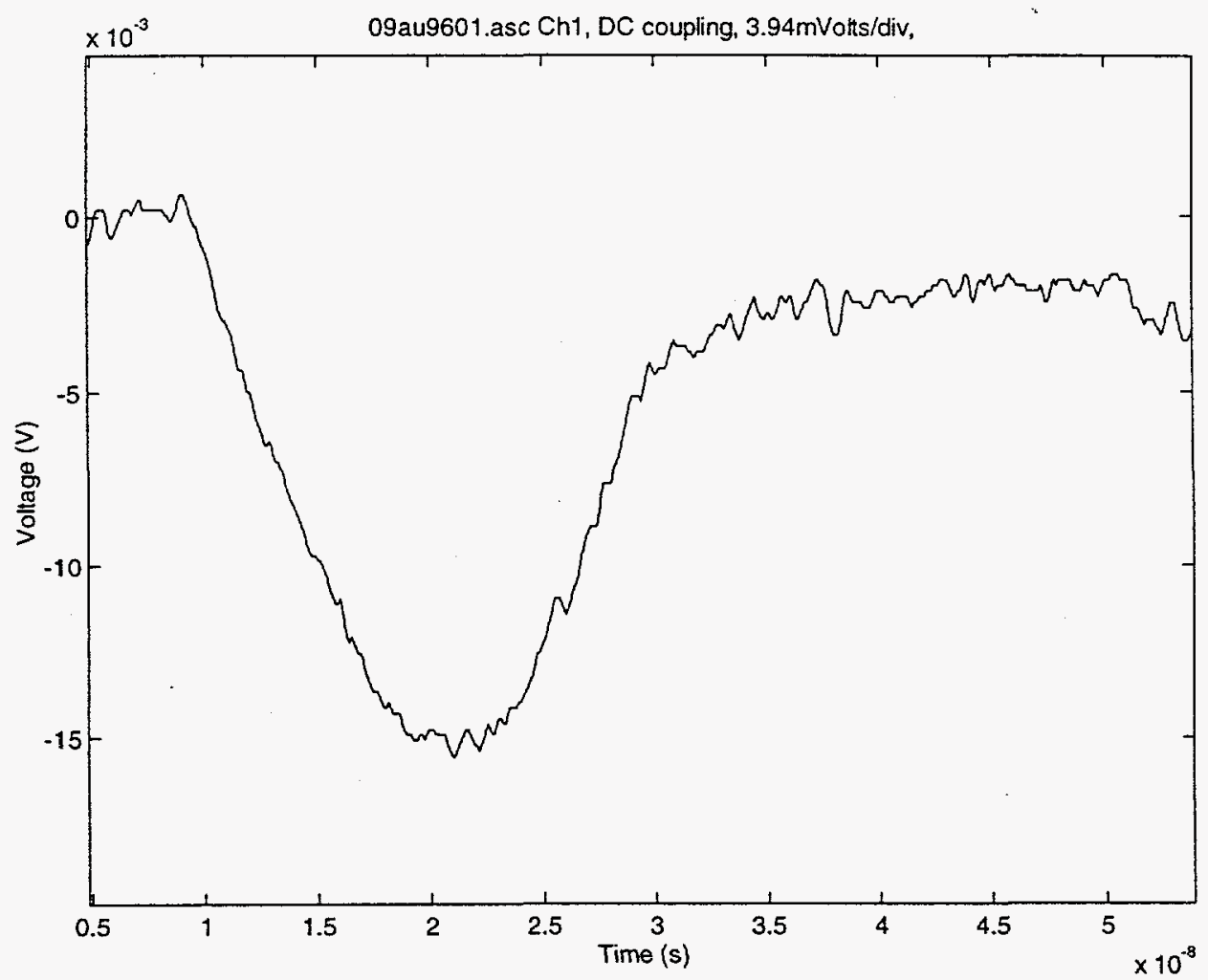

Figure 4.1. Temporal profile of the output from a single laser diode. The output of the laser is delivered through an optical fiber to trigger the switch. The output of the fiber has a total energy of $150 \mathrm{~nJ}$ per pulse. The full width half maximum duration of the pulse is about $15 \mathrm{~ns}$ and the full width is about $30 \mathrm{~ns}$. The power is $5 \mathrm{~W}$. 


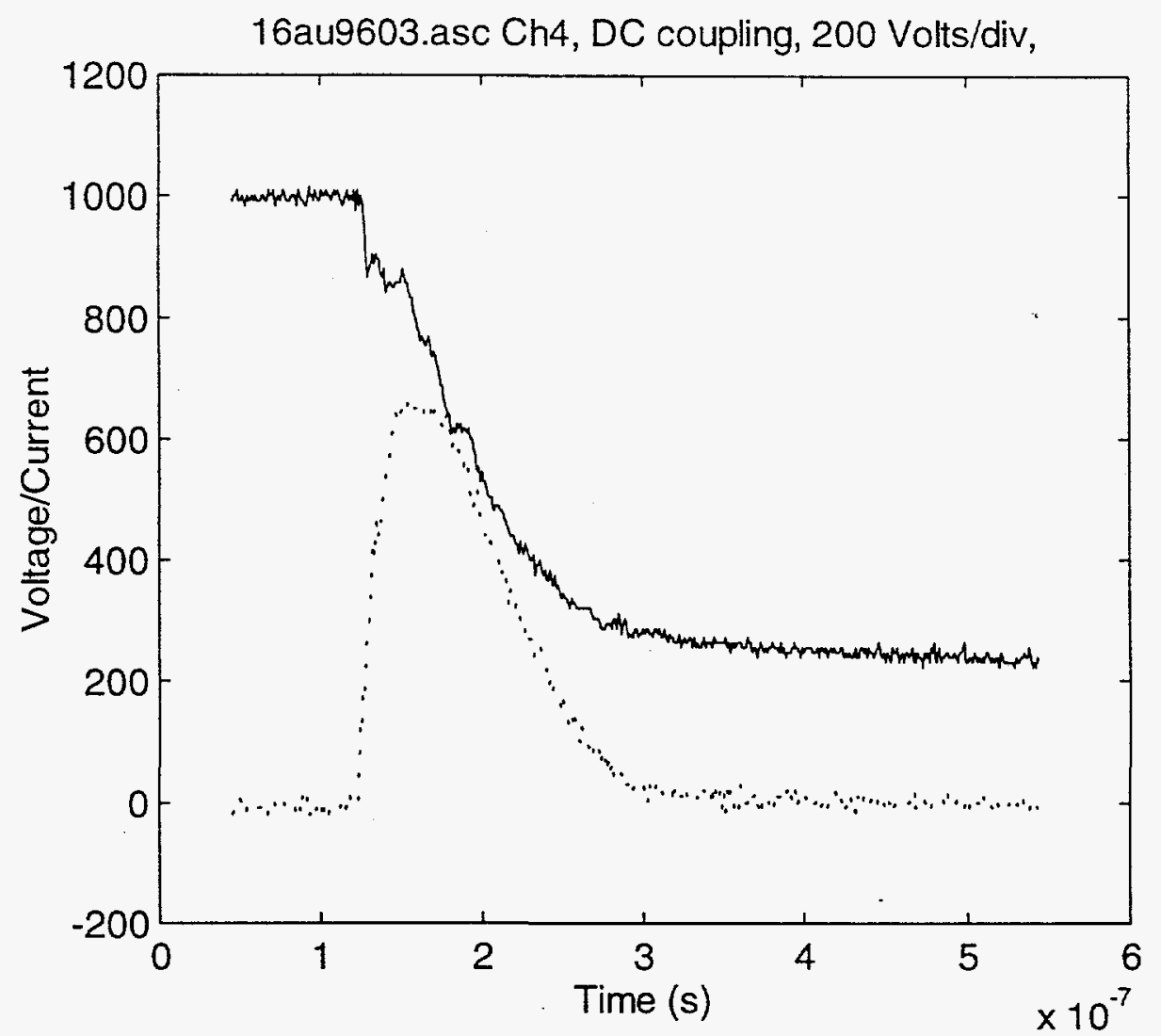

Figure 4.2. The voltage and current waveforms for a high gain PCSS triggered with a single laser diode. The initial voltage is $1 \mathrm{kV}$. Peak current is about $650 \mathrm{~A}$.

\subsection{VERTICAL CAVITY SURFACE EMTTTING LASERS}

VCSELs are smaller and potentially less expensive than the edge emitting lasers in that they can be manufactured and tested in dense 2D arrays without the need for wafer cleaving. They potentially allow integration of the switch and the laser into a single device, using the surface-normal emission of the VCSEL to direct trigger light into the gap of a PCSS. We demonstrated that PCSS can be triggered with high power density optical pulses of reduced energy ( $2 \mathrm{~nJ}$ in a $70 \times 70 \mu \mathrm{m} 2$ square). This represents a significant reduction in system size and cost for applications using PCSS, because these lasers are less expensive, more efficient, and much smaller than those presently being used for triggers. Short-pulse VCSEL and edge emitting semiconductor laser development, which is being pursued by other SNL programs, may soon produce even better lasers to trigger PCSS. Single element edge-emitting laser diodes are being developed which produce higher power and improved beam quality than can be obtained from incoherent laser arrays.

The large energy we use to trigger the switch using laser diode arrays may discourage any attempts to use VCSELs to trigger the switches. Fortunately, the ability of a given laser to 
trigger a high gain GaAs switch depends on many factors such as laser wavelength, energy, pulse duration, illumination geometry, electric field in the switch, etc...One important parameter that we investigated (in February of 1996) to determine if small VCSELs could trigger the switches is brightness. In general, the ability to trigger the switch depends on the initial carrier density created by the laser. Thus, it is not laser energy that is important, but energy per unit volume. For a given wavelength and hence a given penetration depth, the energy per unit volume is proportional to the energy per unit area. To test this concept we used a fiber bundle with fibers of different diameters: $40 \mu \mathrm{m}, 75 \mu \mathrm{m}, 150 \mu \mathrm{m}, 200 \mu \mathrm{m}, 300$ $\mu \mathrm{m}$, and $400 \mu \mathrm{m}$. A Q-switched, frequency doubled, Nd:YAG laser illuminated one end of the fiber bundle with a known energy, while different fibers were used to trigger the switch. The results show that a peak energy per unit area of about $4 \times 10^{-4} \mathrm{~J} / \mathrm{cm}^{2}$ is required to trigger the switch, independent of whether the light was incident over the whole area of the switch or focused to a spot. The best result, in terms of energy, occurred when we triggered the switch with a small area of illumination. In that case we were able to trigger the switch with only 13 $\mathrm{nJ}$ of energy in the fiber (absorbed energy was $8.3 \mathrm{~nJ}$ ), in an area of $12.6 \times 10^{-6} \mathrm{~cm}^{2}$. This experiment gave credibility to an attempt to use VCSELs for triggering the switches.

VCSELs may play an important role in the implementation of an integrated switch/ laser system due to their advantages of surface normal emission and ease of array fabrication and on-wafer testing. It is possible to grow a VCSEL on the back of a switch, isolated from the switch by a passivated layer such that the optical energy from the VCSEL will penetrate the $\mathrm{GaAs}$ wafer and trigger the switch.

In this LDRD we successfully demonstrated the triggering of a PCSS using a VCSEL. As shown in Figure 4.3, this VCSEL emits pulses of $2.15 \mathrm{~nJ}$ energy, and a peak power of 80 $\mathrm{mW}$, values which may be underestimated due to the limited bandwidth of the photodetector and oscilloscope used to obtain the measurement. The laser has a "hollow" square emitting aperture of $70 \mu \mathrm{m}$ width, with a lasing wavelength of $850 \mathrm{~nm}$, and is optimized for high output efficiency (as opposed to low threshold) with only 17 mirror pairs forming the output coupling mirror, and an active region containing 5 quantum wells. Figure 4.4 shows the current and voltage waveforms obtained from the switch triggering. The switch has a $1 \mathrm{~mm}$ gap and was biased at $3.5 \mathrm{kV}$, resulting in a current pulse of $43 \mathrm{~A}$ upon triggering. 


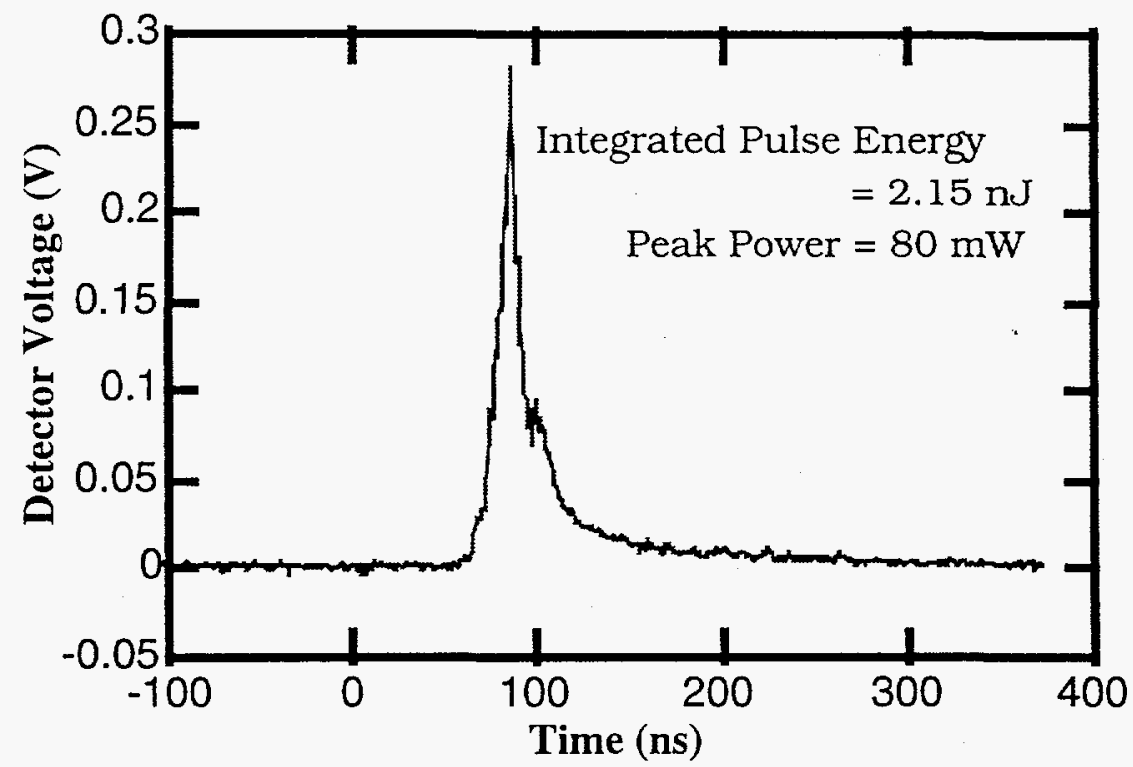

Figure 4.3. The optical output from a VCSEL which was used to trigger a $1 \mathrm{~mm}$ long GaAs PCSS.

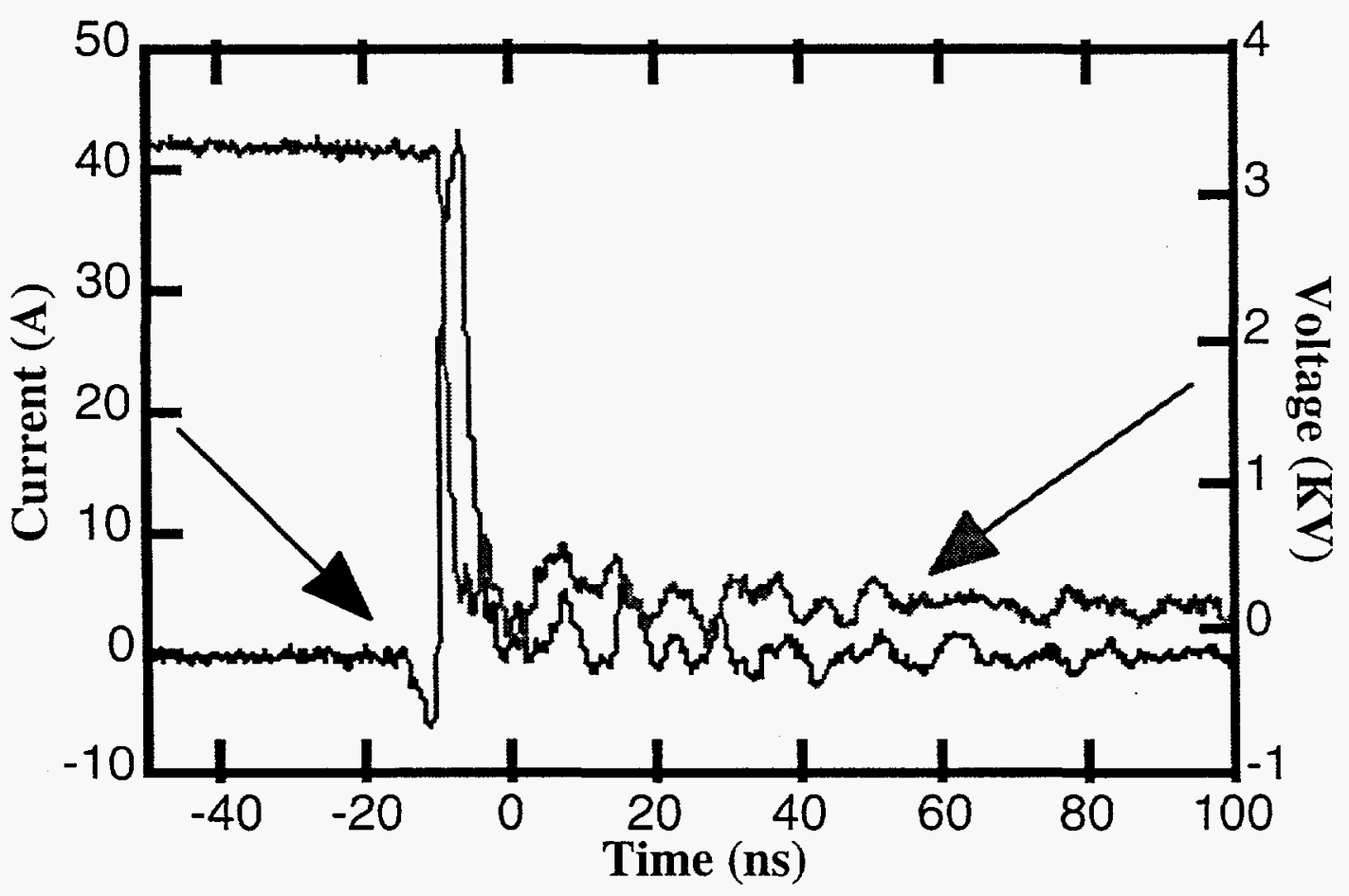

Figure 4.4. Voltage and current from PCSS switch that was triggered with a VCSEL. The switch has a $1 \mathrm{~mm}$ gap and was biased at $3.5 \mathrm{kV}$, resulting in a current pulse of $43 \mathrm{~A}$ upon triggering. 


\subsection{TRANSVERSE JUNCTION STRIPE LASERS}

A fundamental difficulty in laser/switch integration is the semi-insulating (SI) substrate GaAs that PCSS are fabricated on. Most edge-emitting diode laser structures utilize vertical current injection and thus depend on current conduction through the substrate, which is incompatible with PCSS structures. Transverse Junction Stripe (TJS) lasers utilize a lateral lasing junction created typically by dopant diffusion at the wafer surface, resulting in both $\mathrm{p}$ and $\mathrm{n}$-side contacts at the wafer topside. This structure is potentially suitable for fabrication on an SI substrate with PCSS.

In the past, TJS lasers have been demonstrated to be integrable with passive waveguides (Reference 4). Such a structure could be utilized to construct an integrated laser/switch where the TJS laser is fabricated away from the PCSS and a passive waveguide is used to direct the trigger light to the switch gap. The potential advantages for TJS laser/switch integration was the motivation for testing discrete TJS lasers to trigger PCSS. The advantages of integration of TJS lasers with PCSS are three: 1) the TJS is easiest to fabricate on undoped substrates whereas conventional lasers require cladding or an $\mathrm{n}$ type waveguide under the switch, 2) the TJS lasers can be grown to the side of the switch reducing the effect of interference of the switch with the laser when the switch fires, and 3) TJS lasers have been gain switched which increases the laser energy they can produce.

Cleaved facet TJS lasers were tested in similar fashion to the VCSEL lasers to determine their ability to trigger PCSS. As shown in figure 4.5, less pulse energy and peak power were obtained from these particular TIS lasers. The output of the TIS lasers was imaged onto a PCSS, but no triggering was observed. To obtain such triggering, the TJS structure needs to be modified for higher speed and power, but such device development is currently beyond the resources and scope of this program.

Single-element semiconductor lasers are also being fabricated at SNL to produce higher peak power and better beam quality than that of incoherent arrays. Flared waveguide lasers which allow operation at higher peak powers without catastrophic optical damage at the facets have been fabricated and are being tested which so far produce nearly diffraction-limited beam quality with $0.2 \mathrm{~nJ}$ in $29 \mathrm{ps}(7.6 \mathrm{~W})$. Although this energy is small compared to that of incoherent arrays, improvements achieved with these experiments will be scaled to larger lasers. Master oscillator power amplifiers, which utilize a flared laser amplifier stage separate from a gain-switched seed laser will also be fabricated and tested. Devices fabricated this year will also be tested with higher power pulse drive circuitry using PCSS circuits optimized for faster pulses ( $<1 \mathrm{~ns})$. Another issue to address with these experiments is the ability of single heterojunction edge-emitting lasers (SHEEL) give the best gain switching results with PCSS using slower pulses ( $3 \sim 4 \mathrm{~ns}$ ). Double heterojunction and multiquantum well lasers yield better efficiencies and higher peak power than SHEEL when tested with faster pulsers. Speculative ideas on the answer to this question will be tested and the results from these tests will be used to develop more efficient lasers which can produce higher peak powers when driven with PCSS. 


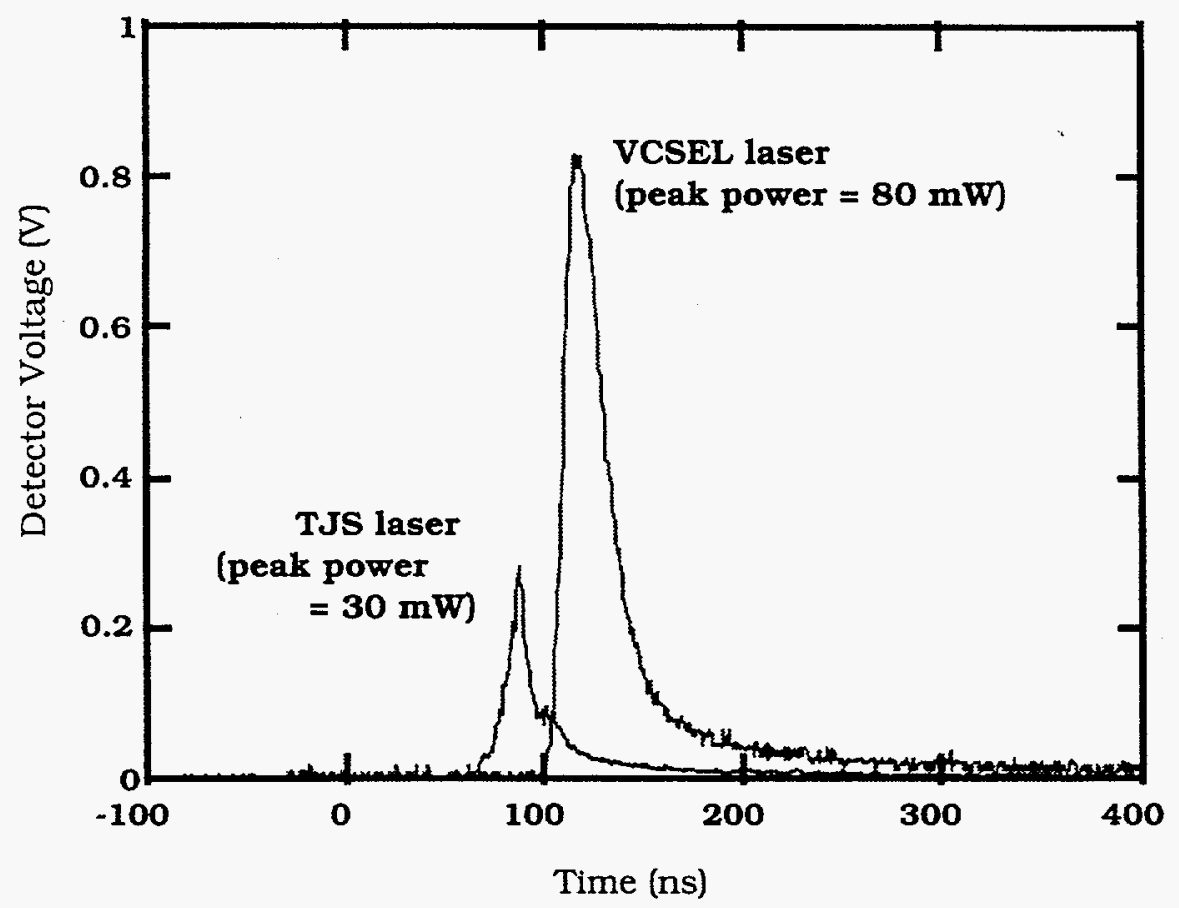

Figure 4.5. The optical output from a VCSEL and a TJS laser. 


\subsection{COMMERCIAL SUPPLIER AND CONCLUSIONS}

We were able use laser diode triggered photoconductive semiconductor switches (PCSS) to activate electro-optic devices such as Q-switches and Pockels cells and we demonstrated that small lasers suitable for laser diode trigger / switch integration did trigger the high gain switches. The technology developed with this LDRD is now the prime candidate for triggering the Q switch in the multiple lasers in the laser trigger system of the X-1 Advanced Radiation Source and may be utilized in other particle beam accelerators. As part of the LDRD we developed a commercial supplier. To study laser/switch integration we tested triggering the high gain GaAs switches with: edge emitting laser diodes, vertical cavity surface emitting lasers (VCSELs), and transverse junction stripe (TJS) lasers. The first two types of lasers (edge emitting and VCSELs) did activate the PCSS but are harder to integrate with the PCSS for a compact package. The TJS lasers, while easier to integrate with the switch, did not trigger the PCSS at the TJS laser power levels we used. The PCSS was used to activate the Q-switch of the compact laser to be used in the X-1 Advanced Radiation Source. 


\subsection{REFERENCES}

1) For general references on high gain PCSS technology see the following:

a.) IEEE Pulsed Power Conferences, 1985-97 (odd years)

b.) IEEE Power Modulator Symposia, 1986-96 (even years)

c.) SPIE Optically Activated Switching Conferences I-IV, 1990, 1992, 1993, 1994.

d.) F. J. Zutavern, G. M. Loubriel, M. W. O'Malley, L. P. Schanwald, W. D. Helgeson, D. L. McLaughlin, and B. B. McKenzie, "Photoconductive semiconductor switch experiments for pulsed power applications," IEEE Trans. Elect. Devices, Vol. 37 , No. 12,1990 , pp. 2472-2477.

e.) A. Rosen and F. J. Zutavern, Eds., High-Power Optically Activated Solid State Switches, Artech House, Boston, 1993, pp. 245-296.

f.) G. M. Loubriel, F. J. Zutavern, H. P. Hjalmarson, R. R. Gallegos, W. D. Helgeson, and M. W. O'Malley, "Measurement of the Velocity of Current Filaments in Optically Triggered, High Gain GaAs Switches", Applied Physics Letters, Vol. 64, No. 24, 13 June, 1994, pp. 3323-3325.

g.) G. M. Loubriel, F. J. Zutavern, A. G. Baca, H. P. Hjalmarson, T. A. Plut, W. D. Helgeson, M. W. O'Malley, M. H. Ruebush, and D. J. Brown, "Photoconductive Semiconductor Switches," IEEE Transactions on Plasma Science, 25, 1997, pp. 124-130.

2) References on switch longevity:

a) G. M. Loubriel, F. J. Zutavern, A. Mar, M. W. O’Malley, W. D. Helgeson, D. J. Brown, H. P. Hjalmarson, and A. G. Baca, "Longevity of Optically Activated, High Gain GaAs Photoconductive Semiconductor Switches," to be published in Proceedings of 11 th IEEE Pulsed Power Conference, Baltimore, MD, June 29- July 2, 1997.

b) G. M. Loubriel, F. J. Zutavern, A. Mar, H. P. Hjalmarson, A. G. Baca, M. W. O'Malley, W. D. Helgeson, D. J. Brown, and R. A. Falk, "Longevity of Optically Activated, High Gain GaAs Photoconductive Semiconductor Switches," to be published in IEEE Transactions on Plasma Science.

3) G. M. Loubriel, F. J. Zutavern, A. G. Baca, H. P. Hjalmarson, W. D. Helgeson, and M. W. O'Malley, "Photoconductive Semiconductor Switches for Firing Sets and ElectroOptic Modulators," Proceedings of 10th IEEE Pulsed Power Conference, Albuquerque, NM, July 10- 13, 1995, pp. 354- 359.

4) G. A. Vawter, J. L. Merz, L. A. Coldren, "Monolithically Integrated Transverse-JunctionStripe Laser with an External Waveguide in GaAs/AlGaAs," IEEE Journal of Quantum Electronics, 25, 1989, pp. 154-162. 


\section{INTERNAL DISTRIBUTION}

\section{DISTRIBUTION}

1313, MS 1060

1314, MS 0603

2674, MS 0328

2674, MS 0328

2674, MS 0328

9000 , MS 0151

9232, MS 0820

9300, MS 1165

9310, MS 1178

9323, MS 1153

9323, MS 1153

9323, MS 1153

9323, MS 1153

9323, MS 1153

9323, MS 1153

9323, MS 1153

9500, MS 1190

9512, MS 1188

9539, MS 1184

9539, MS 1184

9539, MS 1184

9573, MS 1194

4916, MS 0899

8940-2, MS 9018

12690, MS 0619
A. G. Baca

T. E. Zipperian

L. S. Weichman

J. A. Wilder

S. C. Holswade

G. Yonas

H. P. Hjalmarson

J. Polito

J. J. Ramirez

D. W. Brown

M. T. Buttram

W. D. Helgeson

G. M. Loubriel

A. Mar

M. W. O'Malley

F. J. Zutavern

D. L. Cook

R. A. Hamil

D. L. Johnson

J. P. Corley

J. Alexander

D. H. McDaniel

Technical Libary (5)

Central Technical Files

Document Processing for DOE/OSTI (2) 